\title{
Pathological response and outcome after neoadjuvant chemotherapy with DOC (docetaxel, oxaliplatin, capecitabine) or EOF (epirubicin, oxaliplatin, 5-fluorouracil) for clinical T3-T4 non-metastatic gastric cancer
}

\author{
Roberto Petrioli ${ }^{\mathrm{a},{ }^{*}, \text { Daniele Marrelli }}{ }^{\mathrm{b}}$, Franco Roviello ${ }^{\mathrm{b}}$, Alessia D'Ignazio ${ }^{\mathrm{b}}$, Pamela Torre ${ }^{\mathrm{a}}$, \\ Martina Chirra $^{\mathrm{a}}$, Vinno Savelli ${ }^{\mathrm{b}}$, Maria Raffaella Ambrosio ${ }^{\mathrm{c}}$, Guido Francini ${ }^{\mathrm{a}}$, \\ Natale Calomino ${ }^{\mathrm{d}}$, Marco Farsi ${ }^{\mathrm{c}}$, Remo Vernillo ${ }^{\mathrm{e}}$, Edoardo Francini ${ }^{\mathrm{f}, \mathrm{g}}$ \\ ${ }^{\text {a }}$ Medical Oncology Unit, Department of Medicine, Surgery and Neurosciences, University of Siena, Italy \\ ${ }^{\mathrm{b}}$ General Surgery and Surgical Oncology Department, University of Siena, Italy \\ ${ }^{\mathrm{c}}$ Dept Medical Biotechnology, Section of Pathology, University of Siena, Italy \\ ${ }^{\mathrm{d}}$ Clinical Surgery, Department of Surgery and Bioengineering, University of Siena, Siena, Italy \\ ${ }^{\text {e }}$ Clinical Surgery and Surgical Endoscopy, University of Siena, Siena, Italy \\ ${ }^{\mathrm{f}}$ La Sapienza University, Rome, Italy \\ ${ }^{g}$ Oncology Unit, Misericordia Hospital, Grosseto, Italy
}

\section{A R T I C L E I N F O}

\section{Keywords:}

Capecitabine

Gastric cancer

NAC

Neoadjuvant

Oxaliplatin

Docetaxel

5-Fluorouracil

\begin{abstract}
A B S T R A C T
Purpose: In this prospective observational study, we sought to compare the efficacy and safety of docetaxel + oxaliplatin + capecitabine (DOC) with epirubicin + oxaliplatin +5 -fluouracil (EOF) as neoadjuvant chemotherapy (NAC) for clinical T3 or T4 non-metastatic gastric cancer (GC) patients.

Methods: The DOC NAC consisted of docetaxel $35 \mathrm{mg} / \mathrm{m}^{2}$ (days 1-8), oxaliplatin $85 \mathrm{mg} / \mathrm{m}^{2}$ (day 1), and capecitabine $750 \mathrm{mg} / \mathrm{m}^{2}$ twice daily (days 1-14), every 3 weeks. The EOF NAC consisted of intravenous (IV) epirubicin $50 \mathrm{mg} / \mathrm{m} 2$ combined with IV oxaliplatin $130 \mathrm{mg} / \mathrm{m} 2$ on day 1 and continuous infusion 5 -fluouracil 750 $\mathrm{mg} / \mathrm{m} 2$ on days $1-5$, every 3 weeks. After 4 cycles of NAC or upon progression during chemotherapy, patients underwent gastrectomy with standard D2 or D3 lymphadenectomy. Pathological complete response rate per Becker tumor regression grading system was the primary endpoint and the secondary endpoints included progression-free survival (2-yr PFS) and 2-year overall survival (2-yr OS) and tolerability.

Results: Overall, we identified 63 patients with T3-4 non-metastatic GC starting either NAC regimen between January 2010 and December 2017 at our Institution: 34 in the DOC group and 29 in EOF group. Thirty patients $(88 \%)$ in the DOC group and $22(76 \%)$ in the EOF group completed the 4 planned cycles of NAC. Fifty-seven patients received surgery. Results indicated no statistical significant differences between the two groups, and only a trend for some better data in favour of the DOC group. The R0 resection rate was $90.6 \%$ and $88.0 \%$ for the DOC and EOF cohorts, respectively. The pathological complete response rate was $6.2 \%$ in the DOC group and $4.0 \%$ in the EOF group. Becker 1-2 pathological response was found in $46.8 \%$ of the DOC cohort and $28.0 \%$ of the EOF cohort $(\mathrm{p}=.14)$. The 2 -yr PFS rate was $54.1 \%$ for DOC vs. $41.4 \%$ for EOF $(\mathrm{p}=.14)$ and the 2 -yr OS rate was $80.8 \%$ for DOC vs. $58.6 \%$ for EOF $(\mathrm{p}=.05)$. Neutropenia was the most common grade $\geq 3$ toxicity and occurred in $8(23.5 \%)$ patients of the DOC group and $10(34.4 \%)$ patients of the EOF group $(\mathrm{p}=.33)$.

Conclusions: These findings seem to confirm the feasibility of NAC for clinically T3 and T4 non-metastatic GC and, despite no statistical significant difference was documented, suggest a trend for better activity and tolerability for the docetaxel-based regimen (DOC) compared to the epirubicin-based combination (EOF).
\end{abstract}

\footnotetext{
* Corresponding author. Medical Onclogy, University of Siena, Viale Bracci, 53100, Siena, Italy.

E-mail address: r.petrioli@ao-siena.toscana.it (R. Petrioli).
} 


\section{Introduction}

Gastric cancer (GC) is currently the fifth most common malignancy and the third leading cause of cancer mortality, worldwide [1,2]. Although complete surgical resection and adjuvant chemotherapy offer a chance of cure for the localized disease, the prognosis of most patients with clinically T3 or T4 tumors is relatively poor [3,4]. A promising approach for locally advanced GC is a perioperative or neoadjuvant treatment (NAC). The randomized phase 3 MAGIC trial which investigated a perioperative treatment with epirubicin (EPI), cisplatin (CDDP), and 5-fluoruracil (5-FU) (ECF) followed by surgery, demonstrated an improved OS compared with surgery alone for patients with localized or locally advanced inoperable GC [hazard ratio for death (HR), 0.75; 95\% confidence interval (CI), 0.60-0.93; $\mathrm{p}=0.009$ ] [5]. Interesting results, with about $9 \%$ of pathological complete response rate, were also reported in a phase II study with the docetaxel (D) -based DCF (D, CDDP, 5-FU) regimen as perioperative treatment for patients with esophagogastric adenocarcinoma [6]. The frequent observation of severe adverse events, particularly myelotoxicity, nausea/vomiting, and diarrhea, associated with ECF and DCF regimens for advanced gastroesophageal cancer prompted several studies to investigate the efficacy of possible substitutes for the EPI and D partners CDDP and 5FU $[7,8]$. In this regard, oxaliplatin (1-OHP) and capecitabine (Cap) showed similar activity and an improved toxicity profile compared to CDDP and 5-FU, respectively, for patients with advanced GC in randomized clinical trials and thus can be considered suitable replacements for CDDP and 5-FU in the ECF and DCF combination regimens [9,10]. EOF (EPI, 1-HOP and 5-FU) and DOC (D, 1-HOP and Cap) combinations for metastatic gastroesophageal cancer showed encouraging results in terms of objective response rate, progression-free survival (PFS) and overall survival (OS) in several studies [11,12].

Since early 2010, at our Institution NAC was used for patients with clinical T3-T4 non-metastatic GC, with or without lymph node involvement, and initially consisted in the EOF regimen. From 2012 to 2016, our research group participated in the multicenter GastroDOC trial (NCT01876927) which evaluated the DOC combination as perioperative treatment or NAC for patients with locally advanced GC. Based on promising preliminary results (unpublished data), the DOC regimen gradually became the treatment of choice for locally advanced or metastatic GC patients at our institution. However, there is no consensus yet the most effective neoadjuvant regimen for locally advanced GC. Therefore, the aim of this prospective observational study was to compare the efficacy and safety of DOC and EOF combinations as NAC for clinically T3 or T4 GC patients.

\section{Patients and methods}

\subsection{Eligibility criteria}

From the prospectively collected Institutional Review Board approved registry of our Oncology Unit, we selected consecutive patients with histologically proven adenocarcinoma of the stomach with clinical stage non-metastatic T3 or T4, per abdominal computed tomography (CT) scan or laparoscoscopy, who started NAC between January 2010 and December 2017. The other inclusion criteria were age $\geq 18$ years and $\leq 80$ years, Eastern Cooperative Oncology Group (ECOG) performance status (PS) of $0-1$, a life expectancy of at least 3 months, adequate haematological parameters (an absolute neutrophil count of $\geq 1.5 \times 10^{9} / \mathrm{L}$ and a platelet count of $\geq 100 \times 10^{9 /} \mathrm{L}$ ), creatinine and total bilirubin levels $<1.25 \mathrm{x}$ the upper normal limit, aspartate and alanine aminotransferase $<3.0 \mathrm{x}$ the upper normal limit, and absence of a second primary tumor other than non-melanoma skin cancer or in situ cervical carcinoma. Exclusion criteria included severe cardiac dysfunction and chronic diarrhea or uncontrolled sites of infection. The study has been approved by the institutional research ethics committee and has been performed in accordance with the ethical standards as laid down in the 1964 Declaration of Helsinki and its later amendments or comparable ethical standards.

\subsection{Patient evaluation}

The pre-treatment evaluation, performed within 2 weeks before study entry, included a detailed medical history and physical examination, a complete blood cell count, whole-blood chemistry, and computed tomography (CT) scans and/or magnetic resonance imaging (MRI) of the chest and abdomen. During NAC, a complete blood cell count was performed on days 1 and 8 of each cycle. In addition, the patients were clinically assessed at the beginning of each cycle. An echocardiography to monitor the cardiac function was performed within 2 weeks prior to starting and within 2 weeks after completing NAC. Treatment response was evaluated by CT and/or MRI scans per Response Evaluation Criteria in Solid Tumors (RECIST criteria; version 1.1).

\subsection{Treatment delivery}

The DOC NAC regimen consisted of D $35 \mathrm{mg} / \mathrm{m}^{2}$ days $1-8$, combined with $1-$ OHP $85 \mathrm{mg} / \mathrm{m}^{2}$ on day 1 , and Cap $750 \mathrm{mg} / \mathrm{m}^{2}$ twice daily on days $1-14$, every 3 weeks. The EOF NAC combination consisted of intravenous (IV) Epi $50 \mathrm{mg} / \mathrm{m} 2$ combined with IV 1-OHP $130 \mathrm{mg} / \mathrm{m} 2$ on day 1 , and continuous infusion5-FU $750 \mathrm{mg} / \mathrm{m} 2$ days $1-5$, every 3 weeks. To improve tolerability of DOC and EOF regimens for patients $\geq 75$ years, the doses of EPI, 1-HOP and D were reduced by $20 \%$ in the first cycle. Patients in both groups received neoadjuvant treatment for a maximum of 4 cycles.

\subsection{Surgery}

After completing NAC or when progressing during chemotherapy, patients underwent gastrectomy with standard D2 or D3 lymphadenectomy and, when necessary, resection of a small part of the peritoneum or adjacent organs with curative intent.

\subsection{Pathological response}

The cases cohort was represented by formalin-fived and paraffin embedded samples, stored in the archive of Siena University Hospital. All the specimens were processed according to standard automatic procedures. The histologic diagnoses were carried out using the WHO classifications of tumors of the digestive systems and the Lauren classification. The following features were listed in the pathologic report: WHO hystotype, Lauren type, grading, depth of invasion in the wall, vascular and neural infiltration, lymph node status. For the current study all the resection specimens were analysed by two independent local pathologists, and if they disagreed a third pathologist reviewed the slides to reach a majority decision. The tumor regression grading (TRG) system by Becker [13] was used to evaluate pathological response. TRG 1a was defined as complete tumor regression without residual tumor, TRG $1 \mathrm{~b}$ as a subtotal tumor regression with $<10 \%$ residual tumor per tumor bed, TRG 2 is a partial tumor regression with $10-50 \%$ residual tumor, and when residual tumor is $>50 \%$ the tumor regression is classified as TRG 3. This grading system was shown to be associated with prognosis regardless of the postoperative lymph node status [14]. The TNM International Union Against Cancer (UICC) 7th edition classification was used to define the resection status (R0, R1, R2) [15].

\subsection{Toxicity}

The National Cancer Institute Common Terminology Criteria for Adverse Events (NCI-CTCAE; version 4.02) were used to evaluate toxicity. Treatment was delayed if, on the planned day of treatment, the neutrophil count was $<1,500 / \mathrm{mm} 3$, the platelet count was $<100,000$ / $\mathrm{mm} 3$, or the patient had persistent diarrhea or stomatitis $>$ grade 1 . 
Patients who required more than 2 weeks for recovery from adverse reactions were excluded from the study. In case of grade 4 hematological or any other severe ( $\geq$ grade 3 ) non-hematological toxicity, the doses of the chemotherapy agents were reduced by $25 \%$ for the following courses. The dose of 1-OHP was also reduced by $25 \%$ in case of persistent ( $\geq 14$ days) paresthesia or temporary (7-14 days) painful paresthesia or functional impairment. In case of persistent ( $\geq 14$ days) painful paresthesia or functional impairment, l-HOP was omitted from the following cycles until recovery.

Dindo_Clavien classification was used to grade surgical complications [16].

\subsection{Statistical Considerations}

The primary endpoint of this analysis was the pathological response rate per Becker's TRG system. Secondary endpoints were clinical response rate (cRR), calculated as the proportion of patients achieving a complete or a partial remission (PR), R0 resection rate, estimated as the proportion of patients obtaining a R0 resection, 2-year PFS and OS rates, PFS, OS, and safety.

The chi-squared test was used to compare variables between the DOC group and the EOF group. Fisher exact test was used if numbers were $<5$. Two-year PFS (2-yr PFS) rate was defined as the percentage of patients who have not progressed or died from any cause or censored at last follow-up visit, whichever comes first, at 2 years from start of NAC. Twoyear OS (2-yr OS) rate was measured as the portion of patients who have not died or censored at last follow-up visit, at 2 years from NAC onset. The Kaplan-Meier method was used to estimate the distributions of the endpoints, including median time-to-event and its 95\% CI, and the logrank test was used to compare time-to-event distributions between the cohorts. Statistical analyses were performed by MEDCALC software.

\section{Results}

\subsection{Patient characteristics}

Overall, 63 patients were considered eligible for this analysis: 34 in the DOC group and 29 in EOF group. Although no baseline randomisation was performed, patient characteristics were well balanced in terms of sex, ECOG PS, Lauren classification, and clinical stage (Table 1). Median age was 67 years (range 44-80) in the DOC group and 63 years (range 27-76) in the EOF group; there were more patients $>65$ years in DOC group than in EOF group ( $\mathrm{p}=.03)$. The majority of patients was male, with an ECOG PS $=0$, and a poorly differentiated cancer. With the exception of age, the differences between the main baseline characteristics of the two groups were not statistically significant.

Patients had not been stratified by adjuvant chemotherapy yes or no.

\subsection{Clinical response}

Thirty patients (88\%) in the DOC group and $22(76 \%)$ in the EOF group completed the 4 planned cycles of NAC. The cRR was $44.1 \%$ for the DOC cohort and $34.4 \%$ for the EOF cohort $(\mathrm{p}=.43)$ (Table 2). The rate of pT3-4 was $71.8 \%$ and $88.0 \%$ in the groups treated with DOC and EOF, respectively $(\mathrm{p}=.13)$.

The median duration of follow up was 27.7 months (range 4-72) in the DOC group and 21.3 months (range 4-112) in the EOF group. The 2yr PFS rate was $54.1 \%$ (95\% CI36.2\%-71.3\%) for the DOC cohort and $41.4 \%(95 \%$ CI $25.3 \%-60.2 \%)$ for the EOF cohort ( $\mathrm{p}=.14$ ). The 2 -yr OS rate was $80.8 \%$ (95\% CI 66.5\%-93.8\%) in the DOC group and $58.6 \%$ (95\% CI 43.7\%-75.8\%) in the EOF group ( $\mathrm{p}=.05)$. The median PFS was 17.8 months (95\% CI, 14.4-24.7) in the EOF group and not reached in the DOC group (Fig. 1) ( $\mathrm{p}=.09)$. The median OS was 23.8 months (95\% CI 19.7-34.4) in the EOF group and not reached in the DOC group (Fig. 2) $(\mathrm{p}=.07)$.

In subgroup analysis, no statistically significant differences in
Table 1

Patient characteristics.

\begin{tabular}{|c|c|c|}
\hline & DOC & EOF p value \\
\hline Patients & $34(54)$ & $29(46)$ \\
\hline Median age, years (range) & $67(44-80)$ & $63(27-76)$ \\
\hline$>65$ & $21(61.7)$ & $10(34.4) .03$ \\
\hline$\leq 65$ & $13(38.2)$ & 19 (65.5) \\
\hline Sex & & .91 \\
\hline Male & $23(67.6)$ & $20(68.9)$ \\
\hline Female & $11(32.3)$ & $9(31.0)$ \\
\hline ECOG performance status & & .85 \\
\hline 0 & $30(88.2)$ & $26(89.6)$ \\
\hline 1 & $4(11.7)$ & $3(10.3)$ \\
\hline Site of primary tumor & & .17 \\
\hline $1 / 3$ upper & $9(26.4)$ & $10(34.4)$ \\
\hline $1 / 3$ middle & $19(55.8)$ & $12(41.3)$ \\
\hline $1 / 3$ lower & $6(17.6)$ & $7(24.1)$ \\
\hline Histologic differentiation grade & & .12 \\
\hline Low-intermediate & $8(23.5)$ & $12(41.3)$ \\
\hline High & $26(76.4)$ & $17(58.6)$ \\
\hline Lauren classification & & .74 \\
\hline Intestinal & $13(38.2)$ & $12(41.3)$ \\
\hline Diffuse & $15(44.1)$ & $14(48.2)$ \\
\hline Mixed & $6(17.6)$ & $3(10.3)$ \\
\hline Signet ring cells & $8(23.5)$ & $8(27.5) .71$ \\
\hline $\mathrm{cT}$ & & .35 \\
\hline $\mathrm{T} 3$ & $18(52.9)$ & $12(41.3)$ \\
\hline $\mathrm{T} 4 \mathrm{a}$ & $15(44.1)$ & $16(55.1)$ \\
\hline $\mathrm{T} 4 \mathrm{~b}$ & $1(2.9)$ & $1(3.4)$ \\
\hline $\mathrm{cN}$ & & .46 \\
\hline No & $2(2.9)$ & $1(3.4)$ \\
\hline N1 & $16(47.0)$ & 11 (37.9) \\
\hline N2 & $11(32.3)$ & $10(34.4)$ \\
\hline N3 & $5(14.7)$ & $7(24.1)$ \\
\hline Adjuvant chemotherapy & & .29 \\
\hline Yes & $24(70.5)$ & $23(79.3)$ \\
\hline No & $10(29.4)$ & $5(17.2)$ \\
\hline
\end{tabular}

Note: Data are expressed as numbers (\%) except where otherwise noted. Abbreviations: DOC, docetaxel + oxaliplatin + capecitabin; ECOG, Eastern Cooperative Oncology Group; EOF, epirubicin, oxaliplatin, 5-fluouracil; PS, Performance Status.

Table 2

Clinical response.

\begin{tabular}{lll}
\hline & DOC & EOF \\
\hline Enrolled patients & 34 & 29 \\
Clinical response & & \\
PR & $15(44)$ & $10(34.4)$ \\
SD & $17(50)$ & $16(55.1)$ \\
PD & $2(5.8)$ & $3(10.3)$ \\
Median PFS, months $(95 \% \mathrm{CI})$ & not reached & $17.8(14.4-24.7)$ \\
Median OS, months $(95 \% \mathrm{CI})$ & not reached & $23.8(17.7-34.4)$ \\
\hline
\end{tabular}

Note: Data are expressed as numbers (\%) except where otherwise noted. Abbreviation: $\mathrm{CI}=$ Confidence interval.

survival between the two NAC groups were obtained for the main baseline characteristics, A trend for a better PFS and OS was observed for patients with intestinal type compared with diffuse type in the DOC group ( $\mathrm{p}=.07 ; \mathrm{p}=.12)$.

\subsection{Surgical and pathologic response}

Fifty-seven patients received gastrectomy and D2-D3 lymphadenectomy. Four patients ( 3 in the EOF group and 1 in the DOC group) did not proceed to radical surgery as found with extensive peritoneal carcinomatosis or distant metastases. Two patients (1 in DOC and 1 in EOF group) received palliative surgery. A median of 34 (range 19-76) and 32 


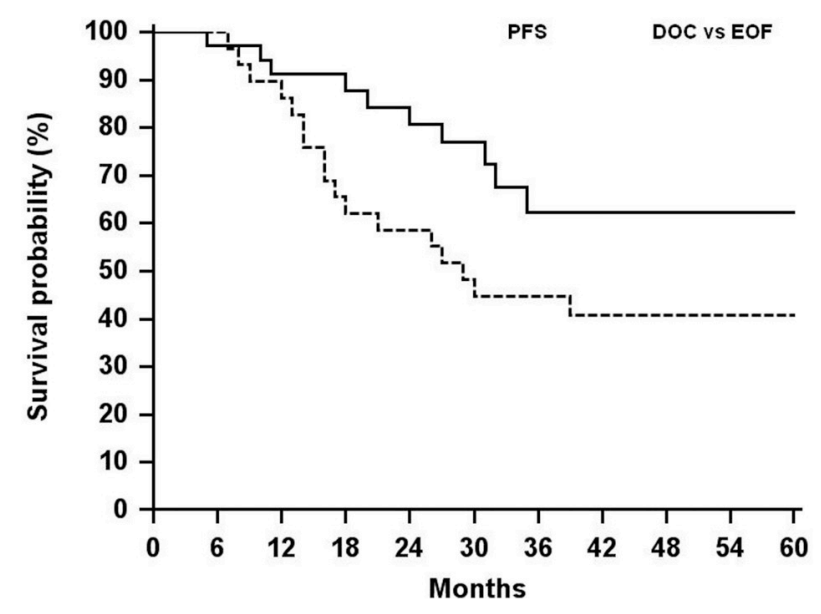

Fig. 1. Progression-free survival of 34 patients in the DOC group (-) and 29 patients in the EOF group (....) $(\mathrm{p}=.09)$.

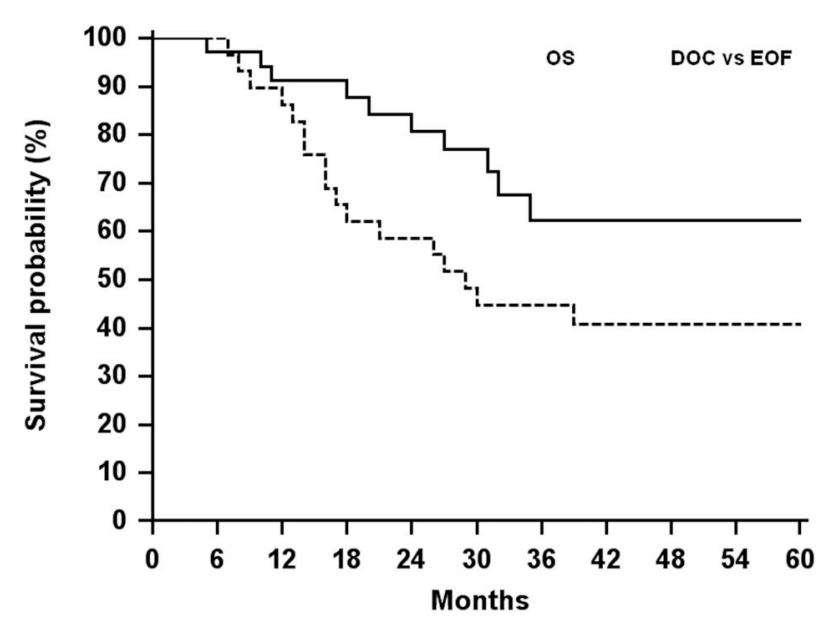

Fig. 2. Overall survival of 34 patients in the DOC group (-) and 29 patients in the EOF group (...) $(\mathrm{p}=.07)$.

(range 18-71) lymph nodes were harvested in DOC and EOF group, respectively.Data of post-surgical complications were recorded for all enrolled patients. Major complications (Dindo_Clavien grade 3-4) arose in 5 patients $(15.6 \%)$ in the DOC group and 3 patients $(12.0 \%)$ in the EOF group $(\mathrm{p}=.69)$. No surgical mortalities were observed.

The $\mathrm{R} 0$ resection rate was $90.6 \%$ in the DOC and $88.0 \%$ in the EOF cohort.

Becker TRG 1a was achieved by 2 patients $(6.2 \%)$ in the DOC group and 1 patient (4.0\%) in the EOF group (Table 3 ) while $46.8 \%$ of subjects receiving DOC and $28.0 \%$ receiving EOF had Becker TRG $1-2$, respectively.

No patient with Becker TRG 1 relapsed in either DOC or EOF group. The survival of patients with Becker 1-2 (complete + partial regression) in DOC group $(n=15)$ and in EOF group $(n=7)$ was compared with survival of patients with Becker TRG 3 (non-responders) in DOC $(n=17)$ and EOF group. A trend for a better PFS and OS was observed for patients with Becker TRG 1-2 with respect to patients with Becker TRG 3 in the DOC group ( $\mathrm{p}=.07 ; \mathrm{p}=.09)$.

In subgroup analysis, only patients with Lauren intestinal type obtained Becker TRG 1 in both NAC treatment groups, and only 6 patients (4 in DOC and 2 in EOF group) with diffuse or signet ring cell carcinoma obtained Becker TRG 2. Complete or partial pathological response was not observed in 5 patients ( 3 in DOC and 2 in the EOF group) with diffuse type located in the lower site of the stomach.
Table 3

Surgical and pathologic response.

\begin{tabular}{lll}
\hline & DOC & EOF p value \\
\hline $\begin{array}{l}\text { Patients } \\
\text { Type of resection }\end{array}$ & 32 & 25 \\
R0 & $29(90.6)$ & .74 \\
R1 & $3(9.3)$ & $22(88.0)$ \\
R2 & 0 & $2(8.0)$ \\
\hline Pathological response - Becker TRG & & $1(4.0)$ \\
1a & $2(6.2)$ & .14 \\
1b & $2(6.2)$ & $1(4.0)$ \\
2 & $11(34.3)$ & $1(4.0)$ \\
3 & $17(53.1)$ & $5(20.0)$ \\
\hline pT & & $18(72.0)$ \\
T0 & $3(9.3)$ & .27 \\
T1 & $1(3.1)$ & $1(4.0)$ \\
T2 & $5(15.6)$ & 0 \\
T3 & $11(34.3)$ & $2(8.0)$ \\
T4 & $12(37.5)$ & $9(36.0)$ \\
\hline pN & & $13(52.0)$ \\
No & $14(43.7)$ & .36 \\
N1 & $7(21.8)$ & $8(32.0)$ \\
N2 & $6(18.7)$ & $4(16.0)$ \\
N3 & $5(15.6)$ & $7(28.0)$ \\
\hline
\end{tabular}

Note: Data are expressed as numbers (\%) except where otherwise noted. Abbreviations: DOC, docetaxel + oxaliplatin + capecitabine; EOF, epirubicin. Oxaliplatin, 5-fluouracil; PD, progressive disease; PR, partial remission. $\mathrm{SD}$, stable disease; TRG, tumor regression grading.

\subsection{Treatment toxicity}

Grade 2 or greater hematologic and non-hematologic adverse events during treatment are shown in Table 4. Neutropenia was the most common grade $\geq 3$ toxicity and occurred in $8(23.5 \%)$ patients treated with DOC and in $10(34.4 \%)$ treated with EOF $(\mathrm{p}=.33)$. Grade 4 neutropenia was observed in 1 patient after 4 EOF cycles. Grade $\geq 3$ anemia and thrombocytopenia were observed in less than $10 \%$ of patients both in the DOC group and the EOF group. None of the patients required platelet or blood red cell transfusions during NAC. Grade 3 or greater stomatitis and nausea/vomiting were lower in the DOC cohort $(8.8 \%$ and $5.8 \%$, respectively) compared to the EOF cohort (20.6\% and $17.2 \%$, respectively) $(\mathrm{p}=.15 ; \mathrm{p}=.17)$. Grade $\geq 3$ neurotoxicity was not observed. No serious hepatic, renal, or cardiac dysfunctions were reported during treatment. During NAC, $21.5 \%$ of DOC cycles and $29.6 \%$ EOF of cycles were delayed due to toxicity and 7 (20.5\%) and 9 (31.0\%) patients required dose-reductions of DOC or EOF, respectively.

Table 4

Grade $\geq 2$ adverse events.

\begin{tabular}{|c|c|c|c|c|}
\hline \multirow{2}{*}{ Adverse event } & \multicolumn{2}{|l|}{ DOC } & \multicolumn{2}{|l|}{ EOF } \\
\hline & Grade 2 & Grade $\geq 3$ & Grade 2 & Grade $\geq 3$ \\
\hline \multicolumn{5}{|l|}{ Hematologic toxicity } \\
\hline Neutropenia & $22(64.7)$ & $8(23.5)$ & $17(58.6)$ & $10(34.4)$ \\
\hline Anemia & $4(11.7)$ & $1(2.9)$ & $5(17.2)$ & $2(6.8)$ \\
\hline Thrombocytopenia & $5(14.7)$ & $2(5.8)$ & $4(13.7)$ & $1(3.4)$ \\
\hline \multicolumn{5}{|l|}{ Non-hematologic toxicity } \\
\hline Nausea/vomiting & $9(26.4)$ & $2(5.8)$ & $10(34.4)$ & $5(17.2)$ \\
\hline Diarrhea & $8(23.5)$ & $3(8.8)$ & $9(31.0)$ & $3(10.3)$ \\
\hline Stomatitis & $8(23.5)$ & $3(8.8)$ & $14(48.2)$ & $6(20.6)$ \\
\hline Hand-foot syndrome & $4(11.7)$ & 0 & $2(6.8)$ & 0 \\
\hline Peripheral neuropathy & $7(20.5)$ & 0 & $4(13.7)$ & 0 \\
\hline Fatigue & $9(26.4)$ & $3(8.8)$ & $6(20.6)$ & $5(17.2)$ \\
\hline
\end{tabular}

Note: Data are expressed as numbers (\%) except where otherwise noted. Abbreviations: DOC, docetaxel + oxaliplatin + capecitabine; EOF, epirubicin, oxaliplatin, 5-fluouracil. 


\section{Discussion}

Currently, there is no standard perioperative or NAC treatment for patients with locally advanced GC. To the best of our knowledge, the present study is the first to compare the D-based regimen DOC with the Epi-based regimen EOF as NAC for patients with T3/4 non-metastatic GC. The vaste majority of patients completed the 4 planned NAC cycles in both treatment groups and achieved a high rate of R0 resection with a low rate of major post-surgical complications; these data seem to indicate the feasibility of both DOC and EOF regimen as NAC in locally advanced GC. A meta-analysis reported that the 2 chemotherapy combinations had similar activity for the treatment of the metastatic disease [17]. In this setting, although no statistical significant differences were observed, our findings suggest a trend for a better efficacy of DOC compared to EOF for locally advanced GC in terms of clinical and pathological response, PFS and OS. Particularly, while the portion of patients achieving a surgical R0 was similar between the 2 combination groups (90.6\% for DOC and 88.0 for EOF), a complete plus partial tumor regression was achieved in almost half of the patients receiving DOC $(46.8 \%)$ versus approximately $1 / 3$ of those having EOF $(28.0 \%)$. Of note, the complete or partial tumor regression was chiefly observed for the intestinal type of GC in both groups, while only 6 patients (4 in DOC and 2 in EOF group) with diffuse or signet ring cell carcinoma obtained a pathological response. Similar findings, albeit in a population with a different stage of disease, were also reported in the large randomized FLOT4-AIO trial of patients with resectable gastric or gastroesophageal cancer treated with perioperative either D- (D, 1-HOP, 5-FU and leucovorin) or Epi-based (Epi, CDDP, 5-FU or Cap) combination chemotherapy. This study reported an increased pathologic response rate, mainly in the intestinal type of cancer, for the D-based regimen compared to that containing Epi [18].

The Becker TRG 1-2 rate observed with the DOC regimen in the current study (46.8\%) was slightly greater than that described in previous studies of different NAC combinations for locally advanced GC, usually less than $40 \%[19,20]$. In contrast, a higher pathological response rate (70\%), combining grade 2 and 3 of the Japanese histologic classification, was obtained with the Epi-containing FLEEOX (5-FU, leucovorin, etoposide, 1-HOP and Epi) regimen in a larger retrospective study [21]. However, the FLEEOX regimen led to a high frequency of grade $3-4$ toxicity.

In addition, a $70 \%$ complete or partial pathologic response rate was reported in a study of patients with locally advanced GC treated with neoadjuvant chemo-radiotherapy followed by D2 gastrectomy [22]. Of note, this was a small retrospective study and the contribution of radiotherapy on the efficacy of this combination is unclear.

In the current study, despite the difference was not statistically significant, a trend for better PFS and OS was observed for patients with Becker TRG 1-2 with respect to patients with Becker TRG 3 in the DOC group ( $\mathrm{p}=.07 ; \mathrm{p}=.09)$. In line with other reports, patients with Becker TRG 1 exhibited the better prognosis, since none of the 6 patients with complete pathological regression relapsed. In this setting, only Becker TRG 1 was found to be associated with better prognosis in some studies, while Becker TRG 2-3 was considered non-responders [14]. Of note, patients with Lauren diffuse type or signet ring cell carcinoma, who were poorly responsive to neoadjuvant treatments in previous reports, rarely obtained complete or pathological response in both treatment groups in the current study [14,23]. However, our results come from a single center study with few patients and definitive conclusions cannot be drawn. Another limitation of the current study was the long accrual period and the non-randomised design. Nevertheless patients were initially allocated in EOF treatment since in 2010 the Epi-based regimen was largely used in advanced GC at our institution. Subsequently, due to favorable results in terms of efficacy and tolerability, the D-based regimen DOC became the treatment of choice both in advanced disease and as NAC.

Although the $\mathrm{p}$ value did not reach the statistical significance, the rates of patients free of progression or alive at 2 years from DOC start were greater than those of patients receiving EOF (54.1\% vs. $41.4 \%$, and $80.8 \%$ vs. $58.6 \%)(\mathrm{p}=.14 ; \mathrm{p}=.05)$. With median OS and PFS that were being reached in the DOC group, probably due to a short median followup, the curves highlight a trend for a better OS and PFS in the cohort having DOC compared that treated with EOF (Figs. 1 and 2) $(\mathrm{p}=.09$; $\mathrm{p}=.07$ ). In this setting, a recent randomised phase $2 / 3$ trial documented an improved OS of the D-based triplet FLOT regimen compared to the Epi-based triplet ECF/EOX regimen (hazard ratio 0.77 ; $95 \%$ CI 0.63 to 0.94 ; median OS 50 months vs 35 months) as perioperative treatment in locally advanced gastric or GEJ adenocarcinoma. Although this large trial suggested a superior efficacy of the D-based regimen FLOT compared to the Epi-based ECF or ECX, serious adverse events were reported in $27 \%$ of patients and hospitalisation for toxicity occurred in $25 \%$ of patients in the FLOT group [24].

In the current study, despite NAC was well tolerated in both treatment groups and the differences were not statistically significant, grade $\geq 3$ neutropenia, stomatitis, and nausea/vomiting were less frequent with DOC compared to EOF. Furthermore, the toxicity we reported for the DOC regimen seemed more favorable than that reported for other Dcontaining combinations used as NAC $[17,25,26]$ In this respect, a lower dose of 1-OHP per cycle $\left(85 \mathrm{mg} / \mathrm{m}^{2}\right)$ in the present study compared to that more commonly used $\left(100 \mathrm{mg} / \mathrm{m}^{2}\right)$, the use of 1-HOP in substitution of CDDP, and the administration of D fractionated on day 1 and 8 as opposed to the more commonly administrating it on day 1 only $\left(60 \mathrm{mg} / \mathrm{m}^{2}\right)$ could partly explain the better tolerability than other DOC or DCF regimens. The feasibility of DOC with the schedule used in the current study seems to be supported also by the lower incidence of patients requiring dose reductions (17.6\%) compared to that observed with other triplet chemotherapy combinations as NAC for gastric and GEJ cancer [17].

In conclusion, in this single-institution study, the D-based regimen (DOC) used as NAC for patients with T3/4 non-metastatic GC showed encouraging results and seemed to produce an advantage in terms of clinical and pathological response, PFS and OS, and safety compared to the Epi-based combination (EOF). Prospective randomized clinical trials on larger samples would be necessary to confirm the greater efficacy and safety of the DOC regimen for patients with locally advanced GC.

\section{Human rights statement}

All procedures followed were in accordance with the ethical standards of the responsible committee on human experimentation (institutional and national) and with the Helsinki Declaration of 1964 and later versions. Informed consent to be included in the study, or the equivalent, was obtained from all patients.

\section{Funding}

This study received no funding.

\section{Declaration of competing interest}

The authors declare that they have no conflict of interest.

\section{Acknowledgments}

We thank the following members of the Multidisciplinary Oncology Group in Gastrointestinal Tumors: Dr. F. Papi, Dr. A. Bernini, Dr. S. Civitelli, Clinical Surgery, University of Siena; Dr. Raffaele Macchiarelli, Dr. Mario Marini, Gastroenterology, University of Siena.

\section{Appendix A. Supplementary data}

Supplementary data to this article can be found online at https://doi. org/10.1016/j.suronc.2019.10.002. 


\section{References}

[1] F. Bray, J. Ferlay, I. Soerjomataram, R.L. Siegel, L.A. Torre, A. Jemal, et al., Global cancer statistics 2018: GLOBOCAN estimates of incidence and mortality worldwide for 36 cancers in 185 countries, CA A Cancer J. Clin. 68 (2018), 394-324.

[2] R.L. Siegel, K.D. Miller, A. Jemal, Cancer statistics, 2019, CA A Cancer J. Clin. 69 (2019) 7-34.

[3] R. Buzzoni, E. Bajetta, M. Di Bartolomeo, R. Miceli, E. Beretta, E. Ferrario, et al., Pathological features as predictors of recurrence after radical resection of gastric cancer, Br. J. Surg. 93 (2006) 205-209.

[4] D. Liu, M. Lu, J. Li, Z. Yang, Q. Feng, M. Zhou, et al., The patterns and timing of recurrence after curative resection for gastric cancer in China, World J. Surg. Oncol. 14 (2016) 305.

[5] D. Cunningham, W.H. Allum, S.P. Stenning, J.N. Thompson, C.J. Van de Velde, M. Nicolson, et al., MAGIC Trial Participants. Perioperative chemotherapy versus surgery alone for resectable gastroesophageal cancer, N. Engl. J. Med. 355 (2006) $11-20$.

[6] L.E. Ferri, S. Ades, T. Alcindor, M. Chasen, V. Marcus, M. Hickeson, et al., Perioperative docetaxel, cisplatin, and 5-fluorouracil (DCF) for locally advanced esophageal and gastric adenocarcinoma: a multicenter phase II trial, Ann. Oncol. 23 (2012) 1512-1517.

[7] E. Van Cutsem, V.M. Moiseyenko, S. Tjulandin, A. Majlis, M. Constenla, C. Boni, et al., Phase III study of docetaxel and cisplatin plus fluorouracil compared with cisplatin and fluorouracil as first-line therapy for advanced gastric cancer: a report of the V325 Study Group, J. Clin. Oncol. 24 (2006) 4991-4997.

[8] A.D. Roth, N. Fazio, R. Stupp, S. Falk, J. Bernhard, P. Saletti, et al., Docetaxel, cisplatin, and fluorouracil; docetaxel and cisplatin; and epirubicin, cisplatin, and fluorouracil as systemic treatment for advanced gastric carcinoma: a randomized phase II trial of the Swiss Group for Clinical Cancer Research, J. Clin. Oncol. 25 (2007) 3217-3223.

[9] D. Cunningham, N. Starling, S. Rao, T. Iveson, M. Nicolson, F. Coxon, et al., Upper gastrointestinal clinical studies group of the national cancer research Institute of the United Kingdom. Capecitabine and oxaliplatin for advanced esophagogastric cancer, N. Engl. J. Med. 358 (2008) 36-46.

[10] S.E. Al-Batran, J.T. Hartmann, S. Probst, H. Schmalenberg, S. Hollerbach, R. Hofheinz, et al., Phase III trial in metastatic gastroesophageal adenocarcinoma with fluorouracil, leucovorin plus either oxaliplatin or cisplatin: a study of the Arbeitsgemeinschaft Internistische Onkologie, J. Clin. Oncol. 26 (2008) $1435-1442$.

[11] S. Cascinu, F. Graziano, S. Barni, R. Labianca, G. Comella, R. Casaretti, et al., A phase II study of sequential chemotherapy with docetaxel after the weekly PELF regimen in advanced gastric cancer. A report from the Italian group for the study of digestive tract cancer, Br. J. Canc. 84 (2001) 470-474.

[12] R. Petrioli, E. Francini, F. Roviello, D. Marrelli, A.I. Fiaschi, L. Laera, et al., Sequential treatment with epirubicin, oxaliplatin and 5FU (EOF) followed by docetaxel, oxaliplatin and 5FU (DOF) in patients with advanced gastric or gastroesophageal cancer: a single-institution experience, Cancer Chemother. Pharmacol. 75 (2015) 941-947.

[13] K. Becker, J.D. Mueller, C. Schulmacher, K. Ott, U. Fink, R. Busch, et al., Histomorphology and grading of regression in gastric carcinoma treated with neoadjuvant chemotherapy, Cancer 98 (2003) 1521-1530.
[14] K. Becker, R. Langer, D. Reim, A. Novotny, C. Meyer zum Buschenfelde, J. Engel, et al., Significance of histopathological tumor regression after neoadjuvant chemotherapy in gastric adenocarcinomas: a summary of 480 cases, Ann. Surg. 253 (2011) 934-939.

[15] S. Edge, D.R. Byrd, C.C. Compton, A.G. Fritz, F. Greene, A. Trotti, AJCC Cancer Staging Handbook, Springer, 2010.

[16] D. Dindo, N. Demartines, P.A. Clavien, Classifications of surgical complications: a new proposal with evaluation in a cohort of 6336 patients and results of a survey, Ann. Surg. 240 (2004) 205-213.

[17] R. Petrioli, G. Roviello, L. Zanotti, F. Roviello, K. Polom, A. Bottini, et al., Epirubicin-based compared with docetaxel-based chemotherapy for advanced gastric carcinoma: a systematic review and meta-analysis, Crit. Rev. Oncol. Hematol. 102 (2016) 82-88.

[18] S.E. Al-Batran, R.D. Hofheinz, C. Pauligk, H.G. Kopp, G.M. Haag, K.B. Luley, et al., Histopathological regression after neoadjuvant docetaxel, oxaliplatin, fluorouracil, and leucovorin versus epirubicin, cisplatin, and fluorouracil or capecitabine in patients with resectable gastric or gastro-oesophageal junction adenocarcinoma (FLOT4-AIO): results from the phase 2 part of a multicentre, open-label, randomised phase 2/3 trial, Lancet Oncol. 17 (2016) 1697-1708.

[19] T. Yoshikawa, M. Sasako, S. Yamamoto, T. Sano, H. Imamura, K. Fujitani, et al., Phase II study of neoadjuvant chemotherapy and extended surgery for locally advanced gastric cancer, Br. J. Surg. 96 (2009) 1015-1022.

[20] T. Kinoshita, M. Sasako, T. Sano, H. Katai, H. Furukawa, A. Tsuburaya, et al., Phase II trial of S-1 for neoadjuvant chemotherapy against scirrhous gastric cancer (JCOG 0002), Gastric Cancer 12 (2009) 37-42.

[21] Y. Li, J. Chen, Q. He, X. Ji, X. Wang, C. Fan, et al., Clinical efficacy of neoadjuvant chemotherapy regimens FLEEOX vs. XELOX in patients with initially unresectable advanced gastric cancer: a propensity score analysis, Oncotarget 8 (2017) 86886-86896.

[22] M.S. Kim, J.S. Lim, W.J. Hyung, Y.C. Lee, S.Y. Rha, K.C. Keum, et al., Neoadjuvant chemoradiotherapy followed by D2 gastrectomy in locally advanced gastric cancer, World J. Gastroenterol. 21 (2015) 2711-2718.

[23] D. Reim, R. Gertler, A. Novotny, K. Becker, C.M. zum Buschenfelde, M. Ebert, et al., Adenocarcinomas of the esophagogastric junction are more likely to respond to preoperative chemotherapy than distal gastric cancer, Ann. Surg. Oncol. 19 (2012) 2108-2118.

[24] S.E. Al-Batran, N. Homann, C. Pauligk, T.O. Goetze, J. Meiler, S. Kasper, et al., Perioperative chemotherapy with fluorouracil plus leucovorin, oxaliplatin, and docetaxel versus fluorouracil or capecitabine plus cisplatin and epirubicin for locally advanced, resectable gastric or gastro-oesophageal junction adenocarcinoma (FLOT4): a randomized, phase 2/3 trial, Lancet 6736 (2019) 32557-32561.

[25] U.D. Bayraktar, S. Bayraktar, P. Hosein, E. Chen, L.G. Koniaris, C.M. Rocha-Lima, et al., Preoperative docetaxel/cisplatin/5-fluorouracil chemotherapy in patients with locally advanced gastro-esophageal adenocarcinoma, Med. Oncol. 29 (2012) 1707-1710.

[26] T. Aoyama, K. Nishikawa, K. Fujitani, K. Tanabe, S. Ito, T. Matsui, et al., Early results of a randomized two-by-two factorial phase II trial comparing neoadjuvant chemotherapy with two and four courses of cisplatin/S-1 and docetaxel/cisplatin/ S-1 as neoadjuvant chemotherapy for locally advanced gastric cancer, Ann. Oncol. 28 (2017) 1876-1881. 